\title{
Assessing ultrastructure of crustacean and insect neuromuscular junctions
}

\author{
H.L. Atwood *, R.L. Cooper ${ }^{1}$ \\ Department of Physiology, MRC Neural Group, University of Toronto, Medical Sciences Building, Toronto, Ontario, M5S 1A8, Canada
}

Accepted 15 December 1995

\begin{abstract}
Motor nerve terminals of arthropods provide excellent models for study of synaptic transmission, and their ultrastructure can be investigated in the same endings from which physiological recordings have been obtained. An experimental procedure for marking a recording site for subsequent ultrastructural analysis is described. The most commonly used procedure for ultrastructural analysis has been serial sectioning and three-dimensional reconstruction. This procedure has the advantage of providing information about the entire nerve terminal, including quantitative information on number, sizes, and relative positions of individual synapses and presynaptic 'active zones'. However, several errors may be generated in the process of viewing the sections and making the reconstruction; these errors can in principle lead to overestimation of synapse and active zone size. The errors become relatively more serious for smaller structures. Procedures for alleviating some of the possible errors are outlined. It is desirable to have additional information from other methods, such as freeze-fracture replication, to guide analysis of reconstructions from serial sections. Combined physiological and ultrastructural analysis of arthropod terminals has shown that each terminal has many small synapses, differing in size and in number of active zones, and that in some terminals, many of the observed synapses have a very low probability of transmission when nerve impulses occur at low frequencies.
\end{abstract}

Keywords: Ultrastructure; Synapse; Active zone; Crustacean; Insect; Neuromuscular

\section{Introduction}

Crustacean and insect neuromuscular junctions have been studied at chemical and structural levels to gain insights into the workings of arthropod motor control, and also as models for the operational mechanisms of synapses in general, since more complex and less accessible central synapses are usually less amenable to experimentation. The attractive features of the arthropod motor nerve terminals are the relatively large size and easy identification of the excitatory (glutamatergic) and inhibitory (GABAergic) peripheral axons, and the opportunities for combined physiological and structural work. Overall organization of crustacean and insect motor nerve terminals is similar at the ultrastructural level (Atwood, 1976; Atwood and

\footnotetext{
* Corresponding author. Tel.: (416) 978-2899; Fax: (416) 978-4940; E-mail: HAROLD@SPINE.MED.UTORONTO.CA

${ }^{1}$ Current address: Sch. Biol. Sci., 100 T.H. Morgan Build., University of Kentucky, Lexington, KY 40506-0225, USA.
}

Cooper, 1994). Although the axons and nerve terminals are relatively large, the individual synapses on the nerve terminals are quite small (Fig. 1) and in this respect resemble most of the synapses found in central nervous systems rather than vertebrate neuromuscular junctions. Since the peripheral synapses of crustaceans and Drosophila show various forms of physiological plasticity, such as short-term and long-term facilitation (Jan and Jan, 1978; Atwood and Wojtowicz, 1986; Zhong and $\mathrm{Wu}, 1991)$, there is good reason to study the fine structure of these synapses to ascertain whether physiological plasticity has a structural basis, as suggested by various studies in mammalian central nervous systems (Greenough, 1984; Black et al., 1990; Weiler et al., 1995). The use of Drosophila affords an opportunity to investigate genetic influences on synaptic structure and function. The advantage of the arthropod systems is that changes in both physiology and structure can be followed for individual, identified neurons, often over long time periods (Lnenicka and Atwood, 1985a,b; Atwood and Lnenicka, 1987). Thus, hypotheses relating structure to function can be tested advantageously in these neurons. 


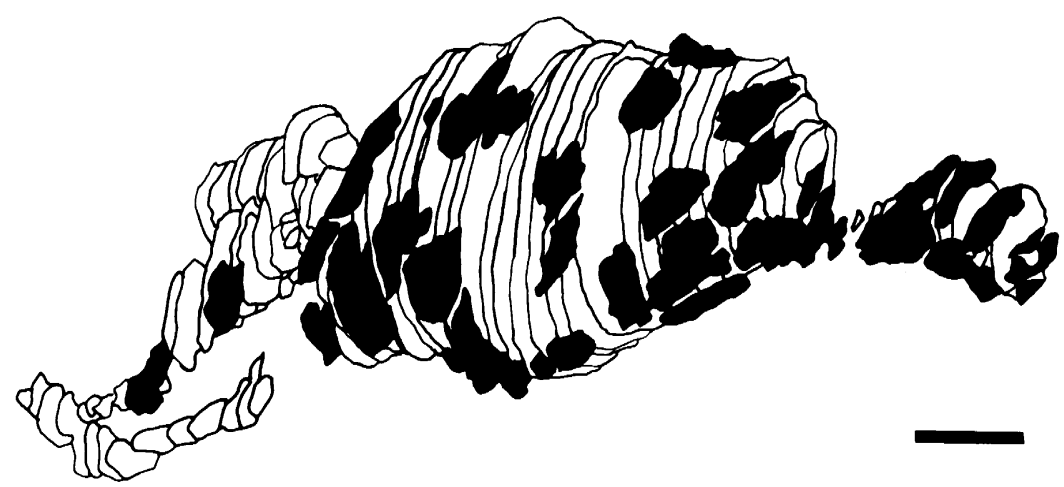

Fig. 1. Reconstruction of a nerve terminal varicosity from serial electron micrographs (crayfish motor axon). Individual synapses are shown. Scale bars: $1.52 \mu \mathrm{m}$.

If one is interested in documenting synaptic size and number for a nerve terminal, a method for resolving the individual synapses is required. To date, the method most often used to determine the number of synapses is reconstruction from serial transmission electron micrographs. (An alternative approach, based upon periodic sampling rather than serial sectioning, employs the 'disector' method, described in detail by Cruz-Orive, 1987). Information from freeze-fracture replicas has also proven useful, but the sample size is small, and it is usually not possible to visualize an entire nerve terminal. It should soon prove possible to employ synapse-specific or vesicle-specific antibodies and confocal microscopy for counting synapses. For example, antibodies to the vesicle-specific protein synaptotagmin indicate the location of synapses in fixed crayfish terminals (Cooper et al., 1995a). Another approach employs vital dyes, such as FM1-43, which are taken up by the nerve during vesicle recycling mechanisms (Betz and Bewick, 1992). These approaches localize, within a broad region, release and recycling events in a nerve terminal, but some synaptic structures are below the limits of resolution for the light microscope, and thus electron microscopy will remain a necessary tool for obtaining high-resolution detail for individual synapses.

Reconstruction of nerve terminals and synapses from serial sections affords detailed information for an individual nerve terminal, but the process is laborious and timeconsuming, and thus the sample size for many studies is rather small. This poses the question of whether the specimens selected for detailed analysis are representative of the population. A larger number of random individual sections can be sampled from various regions to assess this point in purely morphological studies. However, doubt remains on the issue of the adequacy of a morphological sample to address physiological questions. One way to achieve a tighter correlation between physiological and structural studies is to deposit an electron-dense marker from the recording electrode to allow the recording site to

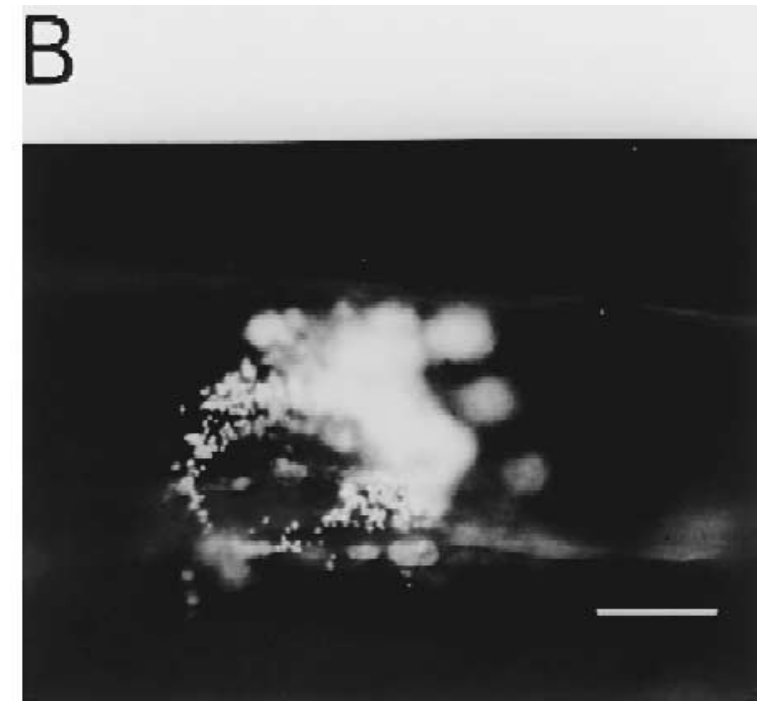

Fig. 2. Method for labelling a nerve terminal varicosity from which recordings of quantal events are made. (A) The recording electrode, with adherent fluorescent polystyrene beads, is placed over a visualized varicosity. (B) The beads adhere to the surface of the tissue, and can be followed subsequently in the resin block and in electron micrographs. Two varicosities are seen within the ring of deposited beads. Scale bar: $30 \mu \mathrm{m}$. 
be subsequently identified for ultrastructural work. This approach has been used successfully in studies of crustacean nerve terminals (Wojtowicz et al., 1994; Cooper et al., 1995c), and could be applied to advantage elsewhere.

\subsection{Correlation of structure and function}

In studies of synaptic strength and plasticity, recordings are often obtained with extracellular 'macro-patch' electrodes placed over a nerve terminal to record the post-synaptic currents generated by transmitter release (Dudel, 1981). Resolution of individual quantal currents is often feasible, and thus the quantal content of transmission (i.e. the number of quantal units generated by a nerve impulse) can be ascertained (Cooper et al., 1995d). The quantal content is thought to be a good indication of the transmitter release from the nerve terminal.

In such recordings, the structures causing the transmitter release have generally not been visualized. With the advent of 'vital' fluorescent dyes, such as 4-Di-2-Asp (Magrassi et al., 1987), it is possible to visualize a living nerve terminal with fluorescence microscopy and to place a macro-patch electrode over a selected part of it (Fig. 2). Electrical recordings can then be made to determine quantal content. If the tip of the electrode has been previously dipped into liquid containing fluorescent polystyrene microspheres $(0.5 \mu \mathrm{m}$ diameter beads are convenient), a fluorescent trace of the edge of the electrode is left at the recording site (Fig. 2). The beads remain on the surface of the tissue as it is processed for electron microscopy, and they are visible within the resin block under fluorescence microscopy (Wojtowicz et al., 1994; Cooper et al., 1995c). The recording site can then be rapidly located by sectioning to the beads, and locating them in thick sections once the site is being approached. Serial thin sections are then cut and checked for the beads, which are readily apparent in transmission electron microscopy. The recording site can then be reconstructed from serial thin sections (Figs. 1 and 2).

Several reconstructions of this type have consistently shown that varicosities from which recordings are made have a substantial number of individual synapses (identified as electron-dense pre- and postsynaptic membranes with a uniform separation of about $20 \mathrm{~nm}$ ). In crayfish terminals, quantal content from a recording site with about 30 synapses is usually $0.5-1$ at low frequencies, suggesting that most synapses are 'silent'. At higher frequencies, quantal content increases (frequency facilitation), indicating recruitment of some silent synapses to the responsive pool (Wojtowicz et al., 1991, 1994). Probably this situation exists in many neuronal systems.

\subsection{Problems in estimating sizes of small structures in serial sections}

In addition to providing counts of the number of synapses on a nerve terminal, the serial sections contain information about the sizes of individual synapses and their associated structures, such as active zone indicators (presynaptic dense bodies: Fig. 3). Attempts have been made in previous work to relate measurements of synaptic or active zone size to synaptic strength (Atwood et al., 1978; Govind and Meiss, 1979; Atwood and Marin, 1983; Govind et al., 1994). Unless one captures an accurate en face view of these structures, or obtains freeze-fracture views of synaptic contacts (Franzini-Armstrong, 1976; Govind et al., 1994), errors in measurement of their size are likely to result from observations of serial sections (Underwood, 1970; Cooper et al., 1995b). The errors may vary in magnitude with size, shape, and orientation of the

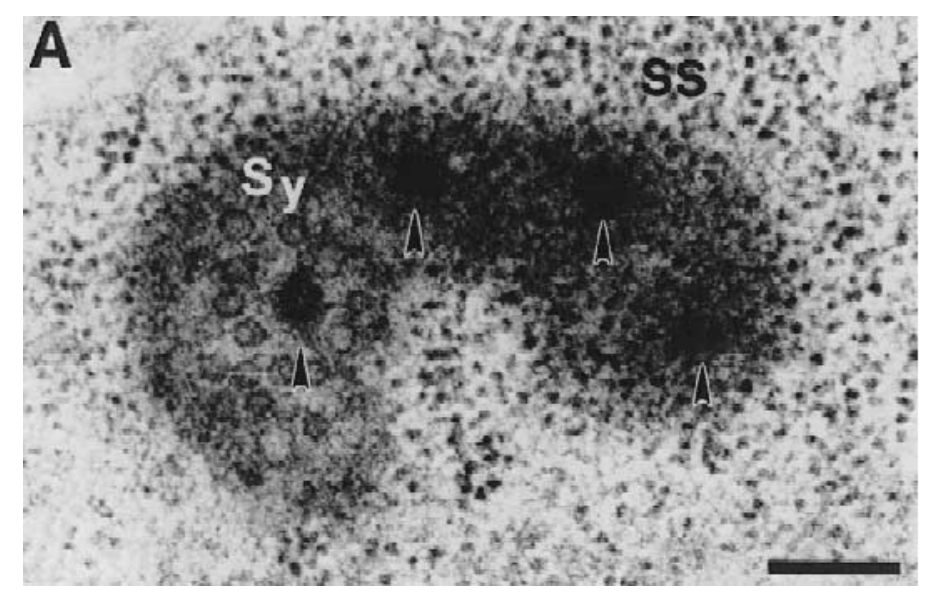

B

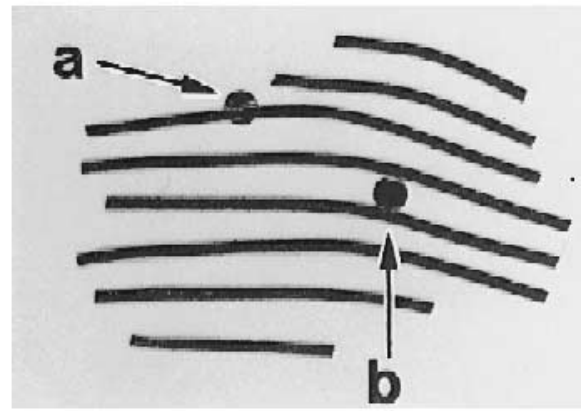

Fig. 3. (A) Electron micrograph of a synapse in tangential section to show closely spaced dense bodies of separate active zones (after Wojtowicz et al., 1994). (B) Reconstruction of an individual synapse possessing closely spaced dense bodies from serial sections. Note discontinuities at the edges of the synapse. Dense bodies are seen in one section (a) or two sections (b). The thick lines represent the synapse mid-way within each section thickness. The distance on either side of the line is the remaining section thickness. The solid dots represent the hemispherical dense bodies observed on the synapse. When the dense dot appears on the line, it was only observed within a single section, whereas if it was observed in two serial sections, it was placed mid-way between them. Scale bar: A, $0.2 \mu \mathrm{m} ; \mathrm{B}, 267 \mathrm{~nm}$. 
structures under investigation. We present below observations on possible errors, some of which can in principle be addressed by corrections.

Additional more detailed discussion of errors in estimation of area and volume of sectioned structures is provided by Cruz-Orive (1987), Cruz-Orive and Weibel (1990), and Baddeley et al. (1986).

\subsubsection{Synaptic area}

Determination of synaptic surface area from serial sections is usually accomplished by measuring the lengths of the synapse (assumed to be cut in the transverse or perpendicular plane) as they are observed in sequential sections of a series, multiplying each length by the section thickness, and adding the resulting areas to get the total area of the synapse (Figs. 1 and 2). This procedure is dependent on stereology of the 3D image projected onto a 2D surface. In some cases, particularly when edges of a synapse are contained in the sections, possible mis-measures can arise. A correction factor can be applied to minimize the resulting over-estimation of synaptic surface area.

It is difficult to measure true synaptic area in small synapses which span only 2-3 sections. Depending on the orientation of a particular synapse to the sectioning plane, the estimates of the surface area can vary. This is illustrated in Fig. 4 with a diamond-shaped synapse oriented in two different directions with respect to the sectioning plane. The synaptic area estimated from the projected 2-D image can vary as much as two-fold. Better estimates of surface area would occur with thinner sections and larger synapses. Thus, the error in measurement depends on section thickness, shape and surface area of the synapse, and orientation of the object in the sectioning plane.

If one assumes that the structures that are seen in the two end sections only project halfway through the section thickness, the measurement error would be reduced and the calculated area would better represent the average area. This may result in underestimating or overestimating the true area in some cases, but it would not always bias the outcome to overestimation as commonly happens with uncorrected measurements. This analysis does neglect the possibility of large infoldings of the edges of the synapse, but in the relatively few cases in which tangential sections have been obtained, large infoldings are not often seen within the thickness of one section. A correction factor, based upon the assumption that the synaptic area occupies half the section thickness in the two end sections in the illustrated diamond-shaped synapses, leads to the estimates shown in Fig. 4. For Fig. $4\left(\mathrm{~A}_{1}\right)$, the corrected estimate provides a value closer to the true value, shown in Fig. 4 $\left(\mathrm{C}_{1}\right)$. In the case of the synapse shown in Fig. $4\left(\mathrm{~A}_{2}\right)$, a correct estimated value is obtained, as shown in Fig. 4 $\left(\mathrm{C}_{2}\right)$.

In order to assess the usefulness of this correction factor, we traced a synaptic area from a tangential section to scale onto a grid of section planes separated by $75 \mathrm{~nm}$, and estimated synaptic area with and without the correction (Fig. 5A). This allows an easy assessment of projected images that would be obtained from sections perpendicular to the synapse. The synapse was then enlarged 2 and 4

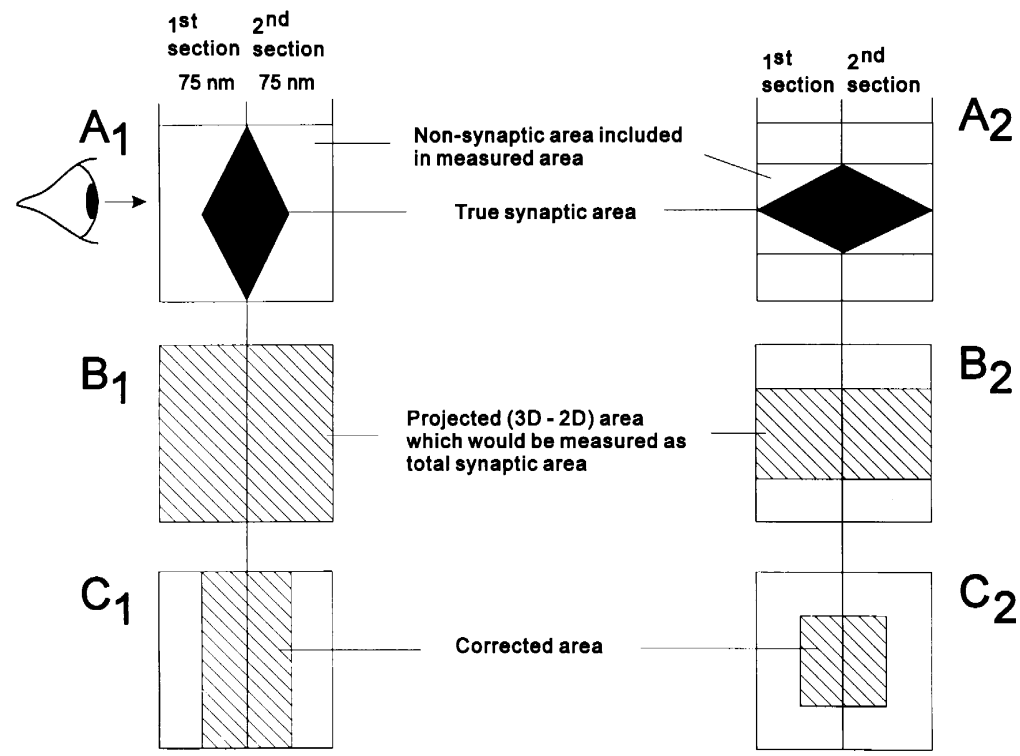

Fig. 4. Determination of synaptic area of a model synapse in serial sections photographed with the electron microscope, illustrating possible errors. (A) For easy representation, a diamond-shaped synapse seen in two successive serial sections is oriented in two ways with respect to the sectioning plane. (B) The projected images illustrate that the estimated area can vary depending on synapse orientation, and that overestimation of the true synaptic area occurs. (C) To approximate, on average, the true synaptic area in the end sections, the observed areas are corrected by assuming that the synapse projects only halfway through the two end sections. Note that without correction $\left(\mathrm{B}_{1}\right)$ the true area is overestimated by 4 times, whereas with the correction $\left(\mathrm{C}_{1}\right)$, the area is only 2 times overestimated. When the synapse is oriented as in $\mathrm{A}_{2}$, the synaptic area is overestimated by a factor of 2 without correction $\left(\mathrm{B}_{2}\right)$, but when the correction is applied, $\left(\mathrm{C}_{2}\right)$, the true area is estimated. 


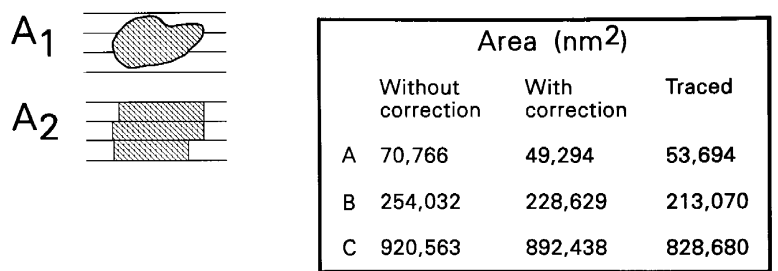

B

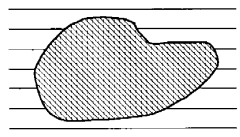

C

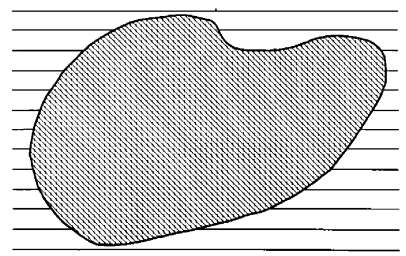

Fig. 5. Effect of synapse size on estimation of area from serial sections. $\left(\mathrm{A}_{1}\right)$ A synapse observed from a tangential section is traced to scale onto a perpendicular sectioning plane of $75 \mathrm{~nm}$. $\left(\mathrm{A}_{2}\right)$ The projected images as seen in electron micrographs, without corrections. $(B, C)$ The same synapse enlarged 2 times (in both $x$ and $y$ axes: B); and 4 times (in both $x$ and $y$ axes: C). The synaptic area was estimated from projected images, as would be done from serial sections of the synapse. The uncorrected and corrected synaptic areas are listed, together with the area determined by digitizing the traced outline area. Note that the projected images produce an overestimation of the area due to curvature of the synaptic edges within all the sections.

times, in both the $x$ and $y$ axes, and remeasured with and without the correction in order to determine the effect of including the correction for larger synapses at the same sectioning thickness (Fig. 5B,C). It is apparent that when the synapse is large, a substantial part of it may lie in the two end sections, which still require the applied correction factor. An additional stereological problem with the sections between the two end sections arises from the progressive curvature of the synapse. The largest width for the synapse within each section will be the observed projected length of the synapse within the section. If the curvature is substantial, the projected images of the serial sections will have step-like discontinuities, and overestimation of total area will occur. The overestimations within each section will be additive and influence the calculated net surface area. To lessen this problem, one can average the observed projected lengths of the synapse in adjacent sections, thus reducing the total calculated surface area. This correction is significant for small synapses, which are common in the vertebrate and invertebrate central nervous system, and can also be significant for large neuromuscular junction synapses, as shown in Fig. 5. For the smallest synapse (Fig. 5A) the errors with and without correction are 8 and $32 \%$, respectively. For the larger synapses (Fig. 5B,C), the errors with correction are 7.3 and $7.7 \%$ respectively, while the errors without correction are 19.2 and $11 \%$, respectively. In this example, larger errors occur for the smaller synapses without correction, while an error of 7-8\% occurs in all cases with correction.

This analysis suggests that measurements from serial reconstructions may, if uncorrected, produce an overestimation of synapse size. However, normal shrinkage of the tissue during fixation and embedding would lead to an error in the opposite direction. If the true size of a synapse is needed, both factors would have to be accounted for in arriving at an accurate estimate. Methods of rapid freezing and freeze substitution have been shown to reduce artifactual distortions due to conventional fixation procedures (Nakajima and Reese, 1983) although such fixation procedures are technically difficult (Heuser and Reese, 1973).

Because the physical relationship between the section plane and the synapse cannot be controlled in the crayfish nerve terminal, serial sectioning of the randomly arrayed synapses over the nerve terminal surface results in some synapses being sectioned between their perpendicular and parallel planes. As a result, projections of synaptic area onto a two-dimensional surface in these orientations biases the results, thus leading to an underestimation of the total, synaptic surface area. In well-oriented preparations, such as the frog neuromuscular junction, the embedded material can be oriented perpendicular to the sectioning plane. Consequently, a surface-area estimation of such a preparation is not subject to this bias. The issue of estimating surface area of structures in relation to the sectioning plane has been previously described in detail (Cruz-Orive and Weibel, 1990; Baddeley et al., 1986).

\subsubsection{Presynaptic dense bodies}

These structures, thought to be a feature of the synaptic active zones in crustaceans and insects, are generally small (spanning 1-2 sections) and often hemispherical in crayfish opener excitatory nerve terminals (Jahromi and Atwood, 1974). In other crustaceans, longer structures often appear, and more complex forms are found in various crustacean and insect species. Since considerable weight has been placed upon the possible correlation between active zone size and transmitter release in various species (Meiss and Govind, 1979; Govind and Meiss, 1979; Walrond and Reese, 1985; Ko and Propst, 1986; Propst et al., 1986; Walrond and Govind, 1987; Propst and Ko, 1987), it is important to assess the accuracy of the size measurements in serial sections.

In a few cases in which tangential sections have been obtained parallel to the surface of a synapse, the true width or diameter of the dense bodies can be measured. The majority of the dense bodies observed in the crayfish opener muscle excitatory terminals appear as hemispheres of $60 \mathrm{~nm}$ diameter sitting on the presynaptic membrane (Fig. 3A). There is a high probability of a $60 \mathrm{~nm}$ diameter hemisphere being sectioned into two observable fragments 

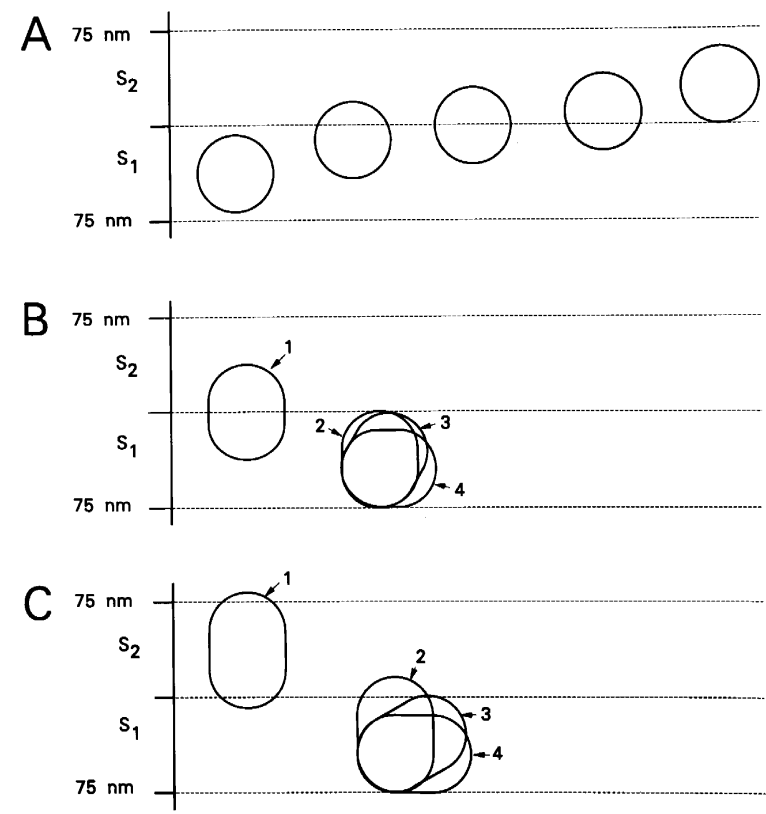

Fig. 6. Determination of the length of synaptic active zone structures (dense bodies) in electron micrographs. (A) Some of the possible orientations of hemispherical dense bodies to the sectioning plane. The projected images of partially sectioned hemispheres can give erroneous interpretation of their true length. (B) A $75 \mathrm{~nm}$ elongated dense bar observed between two sections (1) and at various orientations within a section (2-4). The interpreted length would be $150 \mathrm{~nm}$ in (1), $75 \mathrm{~nm}$ in (2), 70 $\mathrm{nm}$ in (3), and $75 \mathrm{~nm}$ in C4). (C) A $90 \mathrm{~nm}$ elongated dense bar observed between three sections (1) and at various orientations within two sections and one section (2-4). The interpreted length would be 225 in (1), 150 $\mathrm{nm}$ in (2), $85 \mathrm{~nm}$ in (3), and $90 \mathrm{~nm}$ in (4).

when the section thickness is $75 \mathrm{~nm}$ (Fig. 6A). Therefore, dense bodies would often be observed in two sections and could wrongly be assigned a length equivalent to 2 section thicknesses $(150 \mathrm{~nm})$ if it were not known that they are hemispheres (Fig. 6B,C). On occasion, a dense bar is observed in longitudinal orientation that is longer $(>100$ $\mathrm{nm}$ ) than one section thickness (Govind et al., 1994). Sometimes a transversely sectioned dense bar is observed in up to three serial sections. If stereological considerations are not implemented, such structures can be inaccurately estimated in serial sections.

The above considerations have been applied in estimating the sizes and relative positions of parts of dense bodies for individual synapses in crayfish nerve terminals (Fig. 3B). Here, it was assumed that the dense bodies are hemispheres with a diameter of $60 \mathrm{~nm}$, as indicated above. Separation distances were measured from the centers of each dense body.

The interpretation of the length of dense bodies or dense bars varies with section thickness and the angle at which the object lies in the sectioning plane. Three realistic representative cases are given to illustrate this point: a hemispherical dense body $60 \mathrm{~nm}$ in diameter, a dense bar $75 \mathrm{~nm}$ in length, and a dense bar $90 \mathrm{~nm}$ in length (Fig. 6).

A major source of error with length determinations of the elongated dense bars is in estimating the amount of the structure that is present in the two end sections. One cannot assume with any certainty that the objects occupy the entire thickness of both end sections. Only in the middle sections would this occur. The thinner the sections, the more accurate the estimation of size.

An additional problem arises in measuring the projected lengths of objects that are not completely parallel to the sectioning plane. For example, two dense bars $60 \mathrm{~nm}$ in width but of different lengths (75 and $90 \mathrm{~nm}$ ) may be oriented within a section at various angles to the sectioning plane (Fig. 6B,C). The images seen in electron micrographs reveal only the projected lengths of the objects and not their true lengths unless they are sectioned parallel to their long axes. This condition cannot be controlled by the experimenter. Larger deviations in orientation of the dense bar from the parallel arrangement would lead to greater errors in estimating the true length. If the object appears in two sections, the measurement error may increase because in practice one would not know the angle at which the dense bar lies in relation to the sectioning plane nor would one know how far the object penetrates into the thickness of each section.

Estimation of the dense body size and/or volume would be less prone to mathematical error if its physical boundaries were more clearly demarcated. In addition, if there were a structure within the dense body that could be used as an internal reference, one could then estimate the dense body's orientation to the sectioning plane (Cruz-Orive and Weibel, 1990). Since presynaptic dense bodies usually have fuzzy boundaries that vary from 3 to $4 \mathrm{~nm}$ and exhibit no internal structural reference, the best option at present to estimate dimensions from serial-sectioning is to employ the Cavalieri method (Michel and Cruz-Orive, 1988); however, it should be noted that the amount of the structure contained within the two end sections and its true orientation to the sectioning plane are uncertain.

For these reasons, it is desirable to have observations of active zones and dense bodies from tangential sections (Fig. 3) or from freeze-fracture replicas of the presynaptic membrane (Walrond et al., 1993) to guide the assessment of the measurements made from serial sections.

\section{Discussion}

From the serial sectioning approach, a substantial number of papers has been generated on the structure of crustacean and insect motor nerve terminals. These studies have provided much baseline information about the numbers and sizes of synapses in small samples from several different species. In the case of the American lobster (Govind, 1982; Govind et al., 1982; Govind and Pearce, 1982) and a freshwater crayfish (Atwood and Kwan, 1976), a picture has been provided of the changes in synaptic size 
and number for single identified neurons during development and maturation. Synapses become larger and more numerous with time, and change their location as the nerve grows.

Serial sectioning remains the best option for obtaining a complete sample of all synapses on a nerve terminal. In combination with fluorescence microscopy of living nerve terminals and physiological recording methods, this approach provides a means of assessing the relationship between synapse size and number, and physiological effectiveness. Ultrastructural samples can be matched with physiological ones. In addition, preliminary fluorescence microscopy and bead labelling (Fig. 2) allows one to select representative regions of the innervation for ultrastructural work. Recent studies using the combined approach have enhanced the view that many terminals possess silent synapses, and that the number of active synapses can be adjusted during short-term and long-term facilitation to meet physiological requirements (Wojtowicz et al., 1994). In addition, there is reason to believe that the number of synapses on a terminal is only one of the factors responsible for synaptic efficacy in both Drosophila and crustaceans (Kurdyak et al., 1994; Atwood and Cooper, 1994; Cooper et al., 1995c).

As shown here, such studies may be subject to errors affecting the estimates of the sizes of small structures, but the general features of synaptic proliferation and enlargement are not qualitatively altered by these considerations. More detailed work on modeling of 'calcium domains' at synapses as a factor in transmitter release and plasticity (Roberts, 1994) would be highly dependent upon accurate measurements of the sizes of synaptic structures. In view of possible errors in size estimates from serial sections, additional information from freeze-fracture replicas is highly desirable for investigations of the details of synaptic transmission.

As an illustration of a further investigation, we are assessing the separation distances of active zones on single synapses from serial reconstructions. There is a wide range of separation distances for pairs of active zones on individual synapses. These data can be used in a three-dimensional reaction-diffusion model of calcium ions entering at active zones (Winslow et al., 1994b) to determine whether it is likely that the calcium domains from closely associated active zones interact during nerve terminal depolarization (Atwood et al., 1994; Wojtowicz et al., 1994; Cooper et al., 1994, 1996; Winslow et al., 1994a). It is hypothesized that the number of pairs of close active zones could be a structural correlate for the quantal parameter ' $n$ ' (number of participating response elements or 'release sites') observed during low frequencies of stimulation in physiological and stochastic measurements. The corrections outlined in the present paper were applied to optimize the estimates of separation distances between active zones, since modeling at the level of calcium ion diffusion requires precise estimates of spatial relationships of small synaptic structures, including dense bodies, calcium ion channels, and synaptic vesicles.

\section{Acknowledgements}

We thank Dr. Leo Marin for providing electron microscopic technical assistance, as well as Mr. Al Shayan and Ms. M. Hegström-Wojtowicz for additional general assistance. We also thank Mr. Bryan Stewart for fruitful suggestions and Mrs. H.W. Cooper for drawing Fig. 1. This work was supported by a Medical Research Council of Canada and NSERC, Canada grants (H.L.A.) and by a Network of Centres of Excellence (Canada) postdoctoral fellowship (R.L.C.).

\section{References}

Atwood, H.L. (1976) Organization and synaptic physiology of crustacean neuromuscular systems, Prog. Neurobiol., 7: 291-391.

Atwood, H.L. and Cooper, R.L. (1994) Functional and structural parallels in crustacean and Drosophila neuromuscular systems, Am. Zool. 35: $556-565$.

Atwood, H.L. and Kwan, I. (1976) Synaptic development in the crayfish opener muscle, J. Neurobiol., 7: 289-312.

Atwood, H.L. and Lnenicka, G.A. (1987) Role of activity in determining properties of the neuromuscular system in crustaceans, Amer. Zool., 27: 977-989.

Atwood, H.L. and Marin, L. (1983) Ultrastructure of synapses with different transmitter-releasing characteristics on motor axon terminals of a crab, Hyas areneas, Cell Tissue Res., 231: 103-115.

Atwood, H.L. and Wojtowicz, J.M. (1986) Short-term and long-term plasticity and physiological differentiation of crustacean motor synapses, Int. Rev. Neurobiol., 28: 275-362.

Atwood, H.L., Govind, C.K. and Kwan, I. (1978) Nonhomogeneous excitatory synapses of a crab stomach muscle, J. Neurobiol., 9: 17-28.

Atwood, H.L., Cooper, R.L. and Wojtowicz, J.M. (1994) Non-uniformity and plasticity of quantal release at crustacean motor nerve terminals. In L. Stjärne, P. Greengard, S. Grillner, T. Hökfelt and D. Ottoson (Eds.), Molecular and Cellular Mechanisms of Neurotransmitter Release, Raven Press, New York, pp. 363-382.

Betz, W.J. and Bewick, G.S. (1992) Optical analysis of synaptic vesicle recycling at the frog neuromuscular junction, Science, 255: 200-203

Black, J.E., Isaacs, K.R., Anderson, B.J., Alcantara, A.A. and Greenough, W.T. (1990) Learning causes synaptogenesis, whereas motor activity causes angiogenesis, in cerebellar cortex of adult rats, Proc. Natl. Acad. Sci. USA, 87: 5568-5572.

Baddeley, A.J., Gundersen, H.J.G. and Cruz-Orive, L.M. (1986) Estimation of surface area from vertical sections, J. Microsc., 142: 259-276.

Cooper, R.L., Winslow, J.L., Govind, C.K., Pearce, J., Marin, L. and Atwood, H.L. (1994) Structural correlates of quantal parameters at crustacean neuromuscular junction, Abstr. Soc. Neurosci., 20: 550.6.

Cooper, R.L., Hampson, D. and Atwood, H.L. (1995a) Synaptotagmin-like expression in the motor nerve terminals of crayfish, Brain Res. 703: 214-216.

Cooper, R.L., Feuerverger, A., Menzinger, M., Marin, L. and Atwood, H.L. (1995b) Measurement problems associated with the reconstruction of synaptic structures at the electron microscopic level, Abstr. Soc. Neurosci., 21: 709.5 .

Cooper, R.L., Marin, L. and Atwood, H.L. (1995c) Synaptic differentiation of a single motor neuron: conjoint definition of transmitter 
release, presynaptic calcium signals, and ultrastructure, J. Neurosci., 15: 4209-4222.

Cooper, R.L., Stewart, B.A., Wojtowicz, J.M., Wang, S. and Atwood, H.L. (1995d) Quantal measurement and analysis methods compared for crayfish and Drosophila neuromuscular junctions and rat hippocampus, J. Neurosci. Methods, 61: 67-79.

Cooper, R.L., Winslow, J., Govind, C.K. and Atwood, H.L. (1996) Synaptic structural complexity as a factor enhancing probability of calcium-mediated transmitter release, J. Neurophysiol. 75, in press.

Cruz-Orive, L.M. (1987) Particle number can be estimated using a disector of unknown thickness: the selector, J. Microsc., 145: 121-142.

Cruz-Orive, L.M. and Weibel, E.R. (1990) Recent stereological methods for cell biology: a brief survey, Am. J. Physiol., 258: L148-L156.

Dudel, J. (1981) The effect of reduced calcium on quantal unit current and release at the crayfish neuromuscular junction, Pflügers Arch., 391: 35-40.

Franzini-Armstrong, C. (1976) Freeze-fracture of excitatory and inhibitory synapses in crayfish neuromuscular junctions, J. Microsc. Biol. Cell., 25: 217-222.

Govind, C.K. (1982) Development of nerve, muscle and synapse. In D.E. Bliss, H.L. Atwood and D.C. Sandeman (Eds.), The Biology of Crustacea, Vol. 3, Neurobiology: Structure and Function, Academic Press, New York, pp. 185-204.

Govind, C.K. and Meiss, D.E. (1979) Quantitative comparison of lowand high-output neuromuscular synapses from a motoneuron of the lobster (Homarus americanus), Cell Tissue Res., 198: 455-463.

Govind, C.K. and Pearce, J. (1982) Proliferation and relocation of developing lobster neuromuscular synapses, Dev. Biol., 90: 67-78.

Govind, C.K., Meiss, D.E. and Pearce, J. (1982) Differentiation of identifiable lobster neuromuscular synapses during development, J. Neurocytol., 11: 235-247.

Govind, C.K., Pearce, J., Wojtowicz, J.M. and Atwood, H.L. (1994) 'Strong' and 'weak' synaptic differentiation in the crayfish opener muscle: structural correlates, Synapse, 16: 45-58.

Greenough, W.T. (1984) Structural correlates of information storage in the mammalian brain: a review and hypothesis, Trends Neurosci., 7: 229-233.

Heuser, J.E. and Reese, T.S. (1973) Evidence for recycling of synaptic vesicle membrane during transmitter release at the frog neuromuscular junction, J. Cell Biol., 57: 315-344.

Jahromi, S.S. and Atwood, H.L. (1974) Three-dimensional ultrastructure of the crayfish neuromuscular apparatus, J. Cell Biol., 63: 599-613.

Jan, Y.N. and Jan, L.Y. (1978) Genetic dissection of short-term and long-term facilitation at the Drosophila neuromuscular junction, Proc. Natl. Acad. Sci. USA, 75: 515-519.

Ko, C. and Propst, J.W. (1986) Freeze-fracture of physiologically identified neuromuscular junctions from single frog muscle fibers, J. Electron Microsc. Techn., 4: 147-156.

Kurdyak, P., Atwood, H.L., Stewart, B.A. and Wu, C.-F. (1994) Differential physiology and morphology of motor axons to ventral longitudinal muscles in larval Drosophila, J. Comp. Neurol., 350: 463-472.

Lnenicka, G.A. and Atwood, H.L. (1985a) Age-dependent long-term adaptation of crayfish phasic motor axon synapses to altered activity, J. Neurosci., 5: 459-467.
Lnenicka, G.A. and Atwood, H.L. (1985b) Long-term facilitation and long-term adaptation at synapses of a crayfish phasic motoneuron, J. Neurobiol., 16: 97-110.

Magrassi, L., Purves, D. and Lichtman, J.W. (1987) Fluorescent probes that stain living nerve terminals, J. Neurosci., 7: 1207-1214.

Meiss, D.E. and Govind, C.K. (1979) Regional differentiation of neuromuscular synapses in a lobster receptor muscle. J. Exp. Biol., 79: 99-114.

Michel, R.P. and Cruz-Orive, L.M. (1988) Application of the Cavalieri principle and vertical sections method to lung: estimation of volume and pleural surface area, J. Microsc., 150: 117-136.

Nakajima, Y. and Reese, T.S. (1983) Inhibitory and excitatory synapses in crayfish stretch receptor organs studied with direct rapid-freezing and freeze-substitution, J. Comp. Neurol., 213: 66-73.

Propst, J.W. and Ko, C.P. (1987) Correlations between active zone ultrastructure and synaptic function studied with freeze-fracture of physiologically identified neuromuscular junctions, J. Neurosci., 7: 3654-3664.

Propst, J.W., Herrera, A.A. and Ko, C. (1986) A comparison of active zone structure in frog neuromuscular junctions from two fast muscles with different synaptic efficacy, J. Neurocytol., 15: 525-534.

Roberts, W.M. (1994) Localization of calcium signals by a mobile calcium buffer in frog saccular hair cells, J. Neurosci., 14: 3246-3262.

Underwood, E.E. (1970) Quantitative Stereology, Addison-Wesley, Reading, MA.

Walrond, J.P. and Govind, C.K. (1987) Active zone structure at lobster high- and low-output synapses. Soc. Neurosci. Abstr., 13: 317

Walrond, J.P. and Reese, T.S. (1985) Structure of axon terminals and active zones at synapses on lizard twitch and tonic muscle fibers, J. Neurosci., 5: 1118-1131.

Walrond, J.P., Govind, C.K. and Heustis, S. (1993) Two structural adaptations for regulating transmitter release at lobster neuromuscular synapses, J. Neurosci., 13: 4831-4845.

Weiler, I.J., Hawrylak, N. and Greenough, W.T. (1995) Morphogenesis in memory formation: Synaptic and cellular mechanisms, Behav. Brain Res., 66: 1-6.

Winslow, J.L., Cooper, R.L., Govind, C.K., Pearce, J., Marin, L. and Atwood, H.L. (1994a) Close presynaptic active zones may enhance facilitation, Soc. Neurosci. Abstr., 20: 550.5 .

Winslow, J.L., Duffy, S.N. and Charlton, M.P. (1994b) Homosynaptic facilitation of transmitter release in crayfish is not affected by mobile calcium chelators: implications for the residual ionized calcium hypothesis from electrophysiological and computational analyses, J. Neurophysiol., 72: 1769-1793.

Wojtowicz, J.M., Smith, B.R. and Atwood, H.L. (1991) Activity-dependent recruitment of silent synapses, Ann. NY Acad. Sci., 627: 169179.

Wojtowicz, J.M., Marin, L. and Atwood, H.L. (1994) Activity-induced changes in synaptic release sites at the crayfish neuromuscular junction, J. Neurosci., 14: 3688-3702.

Zhong, Y. and Wu, C.-F. (1991) Altered synaptic plasticity in Drosophila memory mutants with a defective cyclic AMP cascade, Science, 251: 198-201. 